\title{
Experimental Concepts for Generating Negative Energy in the Laboratory
}

\author{
E. W. Davis and H. E. Puthoff \\ Inst. for Advanced Studies at Austin, 4030 W. Braker Ln., Ste. 300, Austin, TX 78759, USA \\ 512-342-2187,ewdavis@earthtech.org
}

\begin{abstract}
Implementation of faster-than-light (FTL) interstellar travel via traversable wormholes, warp drives, or other spacetime modification schemes generally requires the engineering of spacetime into very specialized local geometries. The analysis of these via Einstein's General Theory of Relativity (GTR) field equations plus the resultant equations of state demonstrate that such geometries require the use of "exotic" matter in order to induce the requisite FTL spacetime modification. Exotic matter is generally defined by GTR physics to be matter that possesses (renormalized) negative energy density, and this is a very misunderstood and misapplied term by the non-GTR community. We clear up this misconception by defining what negative energy is, where it can be found in nature, and we also review the experimental concepts that have been proposed to generate negative energy in the laboratory.
\end{abstract}

Keywords: Warp Drive, Traversable Wormholes, General Relativity, Squeezed Quantum States, Electromagnetic Field. PACS: 04.20.Cv, 04.20.Gz, 04.62.+v, 42.50.Dv.

\section{INTRODUCTION}

It was nearly two decades ago when science fiction media (TV, film and novels) began to adopt traversable wormholes, and more recently "stargates," for interstellar travel schemes that allowed their heroes and heroines to travel throughout our galaxy. Little did anyone outside of relativity physics know but that in 1985 physicists M. Morris and $\mathrm{K}$. Thorne at CalTech had in fact discovered the principle of traversable wormholes right out of Einstein's General Theory of Relativity (GTR, published in 1915). Morris and Thorne (1988) and Morris, Thorne and Yurtsever (1988) did this as an academic exercise, and in the form of problems for a physics final exam, at the request of Carl Sagan who had then completed the draft of his novel Contact. Sagan wanted to follow the genre of what we call science "faction," whereby the story's plot would rely on cutting-edge physics concepts to make it more realistic and technically plausible. This little exercise ended up becoming one of the greatest cottage industries in general relativity research - the study of traversable wormholes and time machines. Wormholes are hyperspace tunnels through spacetime connecting together either remote regions within our universe or two different universes; they even connect together different dimensions and different times. Space travelers would enter one side of the tunnel and exit out the other, passing through the throat along the way. The travelers would move at $\leq \mathrm{c}(\mathrm{c}=\mathrm{speed}$ of light) through the wormhole and therefore not violate Special Relativity, but external observers would view the travelers as having traversed multi-light year distances through space at FTL speed. A "stargate" was shown to be a very simple special class of traversable wormhole solutions to the Einstein GTR field equations (Visser, 1995; Davis, 2004).

This development was later followed by M. Alcubierre's discovery in 1994 of the "warp drive" spacetime metric, which was another solution to Einstein's GTR field equations. Alcubierre (1994) derived a metric motivated by cosmological inflation that would allow arbitrarily short travel times between two distant points in space. The behavior of the warp drive metric provides for the simultaneous expansion of space behind the spacecraft and a corresponding contraction of space in front of the spacecraft. The warp drive spacecraft would appear to be "surfing on a wave" of spacetime geometry. A spacecraft can be made to exhibit an arbitrarily large apparent FTL speed (>> c) as viewed by external observers, but its moving local rest frame never travels outside of its local comoving light 
cone and thus does not violate Special Relativity. A cottage industry of warp drive spacetimes research ensued and papers were published during the next 15 years.

The implementation of FTL interstellar travel via traversable wormholes, warp drives, or other FTL spacetime modification schemes generally requires the engineering of spacetime into very specialized local geometries. The analysis of these via Einstein's GTR field equations plus the resultant equations of state demonstrate that such geometries require the use of "exotic" matter (or mass-energy) in order to induce the requisite FTL spacetime modification. Exotic matter is generally defined by GTR physics to be matter that possesses (renormalized) negative energy density (sometimes negative stress-tension = positive outward pressure, a.k.a. gravitational repulsion or antigravity), and this is a very misunderstood and misapplied term by the non-GTR community. We clear up this misconception by defining what negative energy is, where it can be found in nature, and we also review the experimental concepts that have been proposed to generate negative energy in the laboratory.

\section{THE GENERAL RELATIVISTIC DEFINITION OF EXOTIC OR NEGATIVE ENERGY}

What is exotic about the matter (or mass-energy) that must be used to generate FTL spacetimes is that it must have negative energy density (Ford and Roman, 2003). The energy density is "negative" in the sense that the configuration of mass-energy we must deploy to generate and thread a traversable wormhole throat or a warp drive bubble must have an energy density ( $\rho_{\mathrm{E}}=\rho c^{2}, \rho=$ mass density) that is less than or equal to its stress $(\tau)$, which is written as: $\rho_{\mathrm{E}} \leq \tau$ (Morris and Thorne, 1988; Visser, 1995). In many cases, the equations of state are also known to possess an energy density that is algebraically negative. It is on the basis of this condition that we call this material property exotic. We will return to the meaning of negative energy within another context in a later section. The condition for ordinary, non-exotic forms of matter that we are all familiar with in nature is that $\rho_{\mathrm{E}}>\tau$. This condition represents one version of what are variously called the weak (WEC), null (NEC), average (AEC), dominant (DEC), and strong (SEC) or "standard" energy conditions (that are mere hypotheses!), which allegedly forbid negative mass-energy density between material objects to occur in nature. Hawking and Ellis (1973) formulated these energy conditions in order to establish a series of mathematical hypotheses governing the behavior of collapsed-matter singularities in their study of cosmology and black hole physics.

However, there are general theorems of differential geometry that guarantee that there must be a violation of one, some or all of the energy conditions (meaning exotic mass-energy is present) for all FTL spacetimes. With respect to creating FTL spacetimes, "negative energy" has the unfortunate reputation of alarming physicists. This is unfounded since all the energy condition hypotheses have been experimentally tested in the laboratory and experimentally shown to be false -25 years before their formulation (Visser, 1990 and references cited therein). For example, the effects of negative energy have been produced in the laboratory via the Casimir Effect and squeezed states of light. Further investigation into this technical issue showed that violations of the energy conditions are widespread for all forms of both classical and quantum matter such as planets, stars, black holes, neutron stars, people, space dust clouds, etc. (Davis, 1999a; Barcelo and Visser, 2002). In addition, Visser (1995) showed that all (generic) spacetime geometries violate all the energy conditions. So the condition that $\rho_{\mathrm{E}}>\tau$ and/or $\rho_{\mathrm{E}}>0$ must be obeyed by all forms of mass-energy in nature is a spurious issue. Violating the energy conditions commits no offense against nature.

\section{Examples of Exotic or "Negative" Energy}

The exotic (energy condition-violating) mass-energy fields that are known to occur in nature are:

- Radial electric or magnetic fields. These are borderline exotic, if their tension were infinitesimally larger, for a given energy density (Herrmann, 1989; Hawking and Ellis, 1973).

- Squeezed quantum states of the electromagnetic field and other squeezed quantum fields; see the following sections for a discussion on squeezed quantum states (Morris and Thorne, 1988; Drummond and Ficek, 2004).

- Gravitationally squeezed vacuum electromagnetic zero-point energy; see the next three sections for the discussion on squeezed quantum states (Hochberg and Kephart, 1991). 
- Casimir Effect energy in flat or curved spaces (DeWitt, 1975; Birrell and Davies, 1984; Saunders and Brown, 1991; Milonni, 1994; Milton, 2001).

- Other quantum fields/states/effects. In general, the local energy density in quantum field theory can be negative due to quantum coherence effects (Epstein, Glaser and Jaffe, 1965). Other examples that have been studied are Dirac field states: the superposition of two single particle electron states and the superposition of two multi-electron-positron states (Vollick, 1998; Yu and Shu, 2003). In the former (latter), the energy densities can be negative when two single (multi-) particle states have the same number of electrons (electrons and positrons) or when one state has one more electron (electron-positron pair) than the other.

Since the laws of quantum field theory place no strong restrictions on negative energies and fluxes, then it might be possible to produce gross macroscopic effects such as warp drive, traversable wormholes, violation of the second law of thermodynamics (Ford, 1978; Davies, 1982), and time machines (Morris, Thorne and Yurtsever, 1988; Visser, 1995; Everett, 1996). The above examples are representative forms of mass-energy that possess negative energy density or are borderline exotic. We will explore most of these in what follows.

\section{Squeezed Quantum States}

Substantial theoretical and experimental work has shown that in many quantum systems the limits to measurement precision imposed by the quantum vacuum zero-point fluctuations (ZPF) can be breached by decreasing the noise in one observable (or measurable quantity) at the expense of increasing the noise in the conjugate observable; at the same time the variations in the first observable, say the energy, are reduced below the ZPF such that the energy becomes "negative." "Squeezing" is thus the control of quantum fluctuations and corresponding uncertainties, whereby one can squeeze the variance of one (physically important) observable quantity provided the variance in the (physically unimportant) conjugate variable is stretched/increased. The squeezed quantity possesses an unusually low variance, meaning less variance than would be expected on the basis of the equipartition theorem. One can in principle exploit quantum squeezing to extract energy from one place in the ordinary vacuum at the expense of accumulating excess energy elsewhere (Morris and Thorne, 1988).

\section{Squeezed Light}

The squeezed state of the electromagnetic field is one of the numerous examples where quantum fields can have negative energy density. Such a state became a physical reality in the laboratory (Slusher et al., 1985; Slusher and Yurke, 1986; Robinson, 1985; 1986) as a result of the nonlinear-optics technique of "squeezing," i.e., of moving some of the quantum-fluctuations of laser light out of the $\cos \omega(\mathrm{t}-\mathrm{z} / \mathrm{c})$ part of the beam and into the $\sin \omega(\mathrm{t}-\mathrm{z} / \mathrm{c})$ part. This pertains to a particular electric field mode of coherent light from an ideal laser: $E=E_{0}[A \cos \omega(t-z / c)+$ $\mathrm{B} \sin \omega(\mathrm{t}-\mathrm{z} / \mathrm{c})]$, where the electric field amplitude $\mathrm{E}_{0}$ is constant, $\omega$ is the angular frequency, $\mathrm{z}$ is the beam propagation direction, $\mathrm{t}$ is time, and (A, B) are the field quadrature operators corresponding to the quantized harmonic oscillator (i.e., the single-mode electromagnetic field) position and momentum, respectively. A and B are defined to be: $\mathrm{A}=\left(a \mathrm{e}^{\mathrm{i} \phi}+a^{\dagger} \mathrm{e}^{-\mathrm{i} \phi}\right) / 2$ and $\mathrm{B}=\left(a \mathrm{e}^{\mathrm{i} \phi}-a^{\dagger} \mathrm{e}^{-\mathrm{i} \phi}\right) / 2 \mathrm{i}$, where $\phi$ is the oscillator phase, and $\left(a, a^{\dagger}\right)$ are the usual annihilation and creation operators. A and B are the observables we are interested in and they must obey the commutation relation: $[\mathrm{A}, \mathrm{B}]=\mathrm{i} \hbar / 2$, where $\hbar$ is Planck's reduced constant. The operators $\left(a, a^{\dagger}\right)$ also obey the commutation relation: $\left[a, a^{\dagger}\right]=1$. And the oscillator vacuum is defined in the usual way: $a|\mathrm{vac}\rangle=0$ (i.e., $a$ annihilates the vacuum state $|\mathrm{vac}\rangle)$. The corresponding uncertainty relation $\Delta \mathrm{A} \Delta \mathrm{B} \geq 1 / 2|\langle[\mathrm{~A}, \mathrm{~B}]\rangle|$ has one built-in degree of freedom: one can "squeeze" the variance of one observable provided the variance in the conjugate observable is stretched. (Note: the $|\langle\ldots\rangle|$ signifies taking the absolute value or magnitude of the expectation value of the enclosed quantity.) Squeezing becomes important when $\Delta \mathrm{A}$ and $\Delta \mathrm{B}$ are limited by the minimized uncertainty product: $\triangle \mathrm{A} \Delta \mathrm{B}=1 / 4$. The observable that gets squeezed will have its fluctuations reduced below the vacuum ZPF. See Drummond and Ficek (2004) for a comprehensive review of the theoretical and experimental state-of-the-art on squeezed light physics. 
To squeeze, one acts on a (complex) state $\chi$ using a "squeeze operator," $\mathrm{S}(\chi)$, for a single quantized harmonic oscillator: $\mathrm{S}(\chi)=\exp \left[1 / 2\left(\chi a^{2}-\chi^{*} a^{\dagger 2}\right)\right]$ (note: $\chi^{*}$ signifies the complex conjugate of $\chi$ ). The state $\chi=r \mathrm{e}^{\mathrm{i} \delta}$ determines the size of the squeezing, where $r$ is the amplitude (giving a measure of the mean photon number in $\chi$ ) and $\delta$ is the phase of squeezing. The "squeezed vacuum" is therefore defined as: $|\chi\rangle=\mathrm{S}(\chi)|\mathrm{vac}\rangle$. Calculating the uncertainties $\Delta \mathrm{A}$ and $\Delta \mathrm{B}$ with respect to the squeezed vacuum gives $\Delta \mathrm{A}=1 / 2 \exp (r)$ and $\Delta \mathrm{B}=1 / 2 \exp (-r)$; therefore $\Delta \mathrm{B}$ is squeezed and $\Delta \mathrm{A}$ is stretched. Thus $\mathrm{S}(\chi)$ reduces the uncertainty in $\mathrm{B}$ and increases that in $\mathrm{A}$ while maintaining the minimized uncertainty product. The act of squeezing transforms the phase space circular noise profile characteristic of the vacuum into an ellipse, whose semimajor and semiminor axes are given by $\Delta \mathrm{A}$ and $\Delta \mathrm{B}$, respectively. This applies to coherent states in general, and the usual vacuum is also a coherent state with eigenvalue zero. As this ellipse rotates about the origin with angular frequency $\omega$, these unequal quadrature uncertainties manifest themselves in the oscillator energy by periodic occurrences, which are separated by one quarter cycle, of both smaller and larger fluctuations compared to the unsqueezed vacuum.

If one squeezes the vacuum, i.e., if one puts vacuum rather than laser light into the input port of a squeezing device, then one gets at the output an electromagnetic field with weaker fluctuations and thus less energy density than the vacuum at locations where $\cos ^{2} \omega(\mathrm{t}-\mathrm{z} / \mathrm{c}) \cong 1$ and $\sin ^{2} \omega(\mathrm{t}-\mathrm{z} / \mathrm{c})<<1$; but with greater fluctuations and thus greater energy density than the vacuum at locations where $\cos ^{2} \omega(\mathrm{t}-\mathrm{z} / \mathrm{c})<<1$ and $\sin ^{2} \omega(\mathrm{t}-\mathrm{z} / \mathrm{c}) \cong 1$ (Caves, 1981; Morris and Thorne, 1988). Since the vacuum is defined to have vanishing energy density, any region with less energy density than the vacuum actually has a negative (renormalized) expectation value for the energy density. Therefore, a squeezed vacuum state consists of a traveling electromagnetic wave that oscillates back and forth between negative energy density and positive energy density, but has positive time-averaged energy density.

\section{Gravitationally Squeezed Electromagnetic ZPF}

A natural source of negative energy comes from the effect that gravitational fields (of astronomical bodies) in space have upon the surrounding vacuum. For example, the gravitational field of the Earth produces a zone of negative energy around it by dragging some of the virtual particle pairs (a.k.a. vacuum ZPF) downward. This concept was initially developed in the 1970s as a byproduct of studies on quantum field theory in curved space (Birrell and Davies, 1982). However, Hochberg and Kephart (1991) derived an important application of this concept to the problem of creating and stabilizing traversable wormholes, and their work was corrected and extended by Davis (1999b). They proved that one can utilize the negative vacuum energy densities, which arise from distortion of the electromagnetic zero point fluctuations due to the interaction with a prescribed gravitational background, for providing a violation of the energy conditions.

Hochberg and Kephart (1991) showed that the squeezed quantum states of quantum optics provide a natural form of matter having negative energy density. The analysis, via quantum optics, shows that gravitation itself provides the mechanism for generating the squeezed vacuum states needed to support stable traversable wormholes. The production of negative energy densities via a squeezed vacuum is a necessary and unavoidable consequence of the interaction or coupling between ordinary matter and gravity, and this defines what is meant by gravitationally squeezed vacuum states. The magnitude of the gravitational squeezing of the vacuum can be estimated from the quantum optics squeezing condition for given transverse momentum and (equivalent) energy eigenvalues (j) of two electromagnetic ZPF field modes, such that this condition is subject to $\mathrm{j} \rightarrow 0$, where $\mathrm{j} \equiv 8 \pi \mathrm{r}_{\mathrm{S}} / \lambda, \lambda$ is the ZPF mode wavelength, and $r_{S}$ is the Schwarzschild radius of the astronomical body (or mass) in question (Hochberg and Kephart, 1991; Davis, 1999b). (Note: $\mathrm{r}_{\mathrm{S}}$ is only a convenient radial distance parameter for each body/mass under examination and there is no black hole collapse involved in this analysis. The Schwarzschild radius is the critical radius, according to GTR, at which a spherically symmetric massive body becomes a black hole, i.e. at which light is unable to escape from the body's surface. We can actually choose any radial distance from the body/mass in question to perform this analysis, but using $\mathrm{r}_{\mathrm{S}}$ makes the equations simpler in form.) This squeezing condition simply states that substantial gravitational squeezing of the vacuum occurs for those ZPF field modes with $\lambda \geq 8 \pi \mathrm{r}_{\mathrm{S}}$ of the mass in question (whose gravitational field is squeezing the vacuum).

The general result of the gravitational squeezing effect is that as the gravitational field strength increases, the negative energy zone (surrounding the mass) also increases in strength. Table 1 shows when gravitational squeezing becomes important for example masses. The table shows that in the case of the Earth, Jupiter and the Sun, this 
squeeze effect is extremely feeble because only ZPF mode wavelengths above $0.2 \mathrm{~m}-78 \mathrm{~km}$ are affected. For a solar mass black hole (radius of $2.95 \mathrm{~km}$ ), the effect is still feeble because only ZPF mode wavelengths above $78 \mathrm{~km}$ are affected. But also note in the table that Planck mass objects will have an enormously strong negative energy zone surrounding them because all ZPF mode wavelengths above $8.50 \times 10^{-34}$ meters will be squeezed, in other words, all wavelengths of interest for vacuum fluctuations. Protons will have the strongest negative energy zone in comparison because the squeezing effect includes all ZPF mode wavelengths above $6.50 \times 10^{-53}$ meters. Furthermore, a body smaller than a nuclear diameter $\left(\approx 10^{-16} \mathrm{~m}\right)$ and containing the mass of a mountain $\left(\approx 10^{11} \mathrm{~kg}\right)$ has a fairly strong negative energy zone because all ZPF mode wavelengths above $10^{-15}$ meters will be squeezed.

TABLE 1. Substantial Gravitational Squeezing Occurs for Electromagnetic ZPF When $\lambda \geq 8 \pi \mathrm{r}_{\mathrm{S}}$ (Davis, 1999b).

\begin{tabular}{c|c|c}
\hline Mass of body $(\mathbf{k g})$ & Schwarzschild radius of body, $\mathbf{r}_{\mathbf{S}}(\mathbf{m})$ & ZPF mode wavelength, $\boldsymbol{\lambda}(\mathbf{m})$ \\
\hline Sun $=2.00 \times 10^{30}$ & $2.95 \times 10^{3}$ & $\geq 78.0 \times 10^{3}$ \\
\hline Jupiter $=1.90 \times 10^{27}$ & 2.82 & $\geq 74$ \\
\hline Earth $=5.98 \times 10^{24}$ & $8.87 \times 10^{-3}$ & $\geq 0.23$ \\
\hline Typical mountain $\approx 10^{11}$ & $\approx 10^{-16}$ & $\geq 10^{-15}$ \\
\hline Planck mass $=2.18 \times 10^{-8}$ & $3.23 \times 10^{-35}$ & $\geq 8.50 \times 10^{-34}$ \\
\hline Proton $=1.67 \times 10^{-27}$ & $2.48 \times 10^{-54}$ & $\geq 6.50 \times 10^{-53}$ \\
\hline
\end{tabular}

We are presently unaware of any way to artificially generate gravitational squeezing of the vacuum in the laboratory. This will be left for future investigation.

\section{A Moving Mirror}

Negative energy can be created by a single moving reflecting surface (a moving mirror), see Figure 1. A mirror moving with increasing acceleration generates a flux of negative energy that emanates from its surface and flows out into the space ahead of the mirror (Birrell and Davies, 1984; Walker, 1985). However, this effect is known to be exceedingly small, and it is not the most effective way to generate negative energy for our purposes. We will not consider this scheme any further.

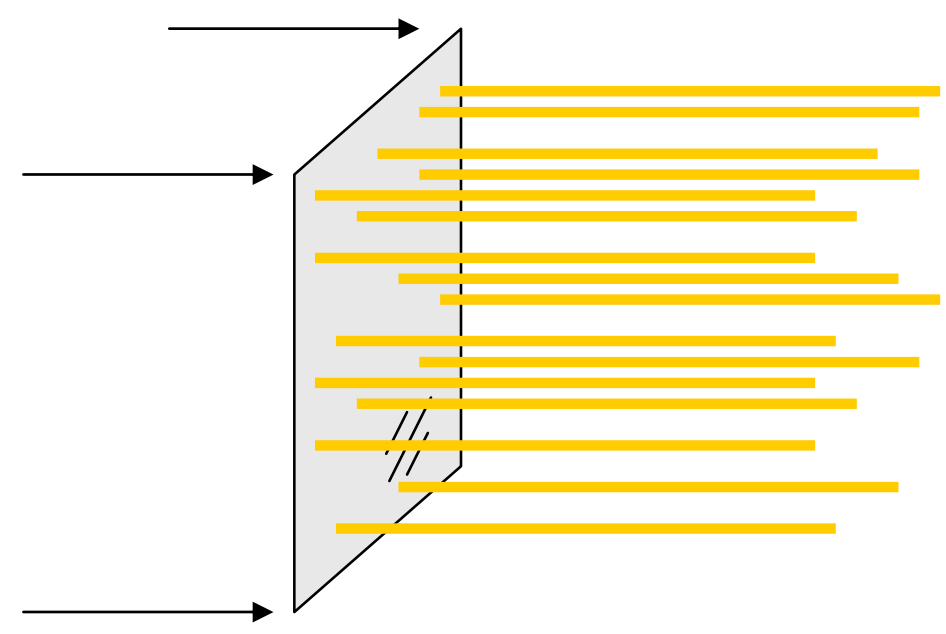

FIGURE 1. Negative Energy Flux (gold) Emanating from a Moving Mirror. 


\section{PROPOSED EXPERIMENTS FOR GENERATING NEGATIVE ENERGY}

In what follows we outline five proposed experiments for generating negative energy in the lab. A detailed technical/engineering analysis of each of these is the subject of future work, and space limits the amount of technical detail that can be provided in this paper.

\section{Negative Casimir Energy}

The Casimir Effect is by far the easiest and most well known way to generate negative energy in the lab. The energy density $\rho_{\mathrm{CE}}=-\left(\pi^{2} \hbar \mathrm{c} / 240\right) \mathrm{d}^{-4}$ (in $\left.\mathrm{J} / \mathrm{m}^{3}\right)$ within a Casimir cavity is negative and manifests itself by producing a force of attraction between the cavity walls ( $\mathrm{d}$ is the wall separation). This has been measured in the lab to a very high level of precision for a number of different cavity geometries (see Milton, 2001 and the references cited therein). But cavity dimensions must be made exceedingly small in order to generate a significant amount of negative energy for our purposes. In order to use the Casimir Effect to generate a spherically symmetric traversable wormhole throat of radius $r_{w h}$, we will need to design a cavity of perfectly conducting spherically concentric thin plates with a plate separation of (Morris, Thorne and Yurtsever, 1988):

$$
\begin{aligned}
d & =\left(\pi^{3} / 30\right)^{1 / 4}\left(r_{w h} \sqrt{\hbar G / c^{3}}\right)^{1 / 2}, \\
& \left.\approx\left(4.05 \times 10^{-18}\right) r_{w h}^{1 / 2} \quad \text { (meters }\right)
\end{aligned}
$$

where $G$ is the Universal Gravitation Constant and $r_{w h}$ is in meters. Equation (1) shows that a $1 \mathrm{~km}$ radius throat will require a cavity plate separation of $1.28 \times 10^{-16} \mathrm{~m}$ (smaller than a nuclear diameter), and the energy density $\left(\rho_{\mathrm{EC}}\right)$ generated by this configuration is $-4.83 \times 10^{36} \mathrm{~J} / \mathrm{m}^{3}$. In contrast, a wormhole with a throat radius of $1 \mathrm{AU}$ (mean Earth-Sun distance) will require a plate separation of $1.57 \times 10^{-12} \mathrm{~m}(35 \%$ smaller than the electron's Compton wavelength), and generate $-2.16 \times 10^{20} \mathrm{~J} / \mathrm{m}^{3}$ of energy density. There is no technology known today that can engineer a cavity with such miniscule plate separations. In addition, such miniscule plate separations are unrealistic because the Casimir Effect switches over into the non-retarded field behavior $\left(\sim \mathrm{d}^{-3}\right)$ of van der Waals forces when plate separations go below the wavelength $(\approx 15 \mathrm{~nm})$ where they are no longer perfectly conducting (Forward, 1983). We will consider this scheme no further.

\section{Radial Electric/Magnetic Fields}

It is beyond the scope of this paper to include all the technical configurations by which one can generate radial electric or magnetic fields. Suffice it to say that ultrahigh-intensity tabletop lasers have been used to generate extreme electric and magnetic field strengths in the lab. Ultrahigh-intensity lasers use the chirped-pulse amplification (CPA) technique to boost the total output beam power. All laser systems simply repackage energy as a coherent package of optical power, but CPA lasers repackage the laser pulse itself during the amplification process. In typical high-power short-pulse laser systems, it is the peak intensity, not the energy or the fluence, which causes pulse distortion or laser damage. However, the CPA laser dissects a laser pulse according to its frequency components, and reorders it into a time-stretched lower-peak-intensity pulse of the same energy (Perry, 1996; 2000; Mourou, Barty and Perry, 1998). This benign pulse can then be amplified safely to high energy, and then only afterwards reconstituted as a very short pulse of enormous peak power - a pulse which could never itself have passed safely through the laser system (see Figure 2). Made more tractable in this way, the pulse can be amplified to substantial energies (with orders of magnitude greater peak power) without encountering intensityrelated problems.

The extreme output beam power, fields and physical conditions that have been achieved by ultrahigh-intensity tabletop lasers are (Mourou, Barty and Perry, 1998):

- Power Intensity $\approx 10^{15}-10^{26}$ Watts $/ \mathrm{cm}^{2}\left(10^{30} \mathrm{~W} / \mathrm{cm}^{2}\right.$ using SLAC as a booster $)$ 
- Peak Power Pulse $\leq 10^{3}$ femtoseconds

- E-fields $\approx 10^{14}-10^{18} \mathrm{~V} / \mathrm{m}$ [note: the critical quantum-electrodynamical (vacuum breakdown) field strength is $\mathrm{E}_{\mathrm{c}}=2 \mathrm{~m}_{\mathrm{e}}{ }^{2} \mathrm{c}^{3} / \hbar \mathrm{e} \approx 10^{18} \mathrm{~V} / \mathrm{m} ; \mathrm{m}_{\mathrm{e}}$ and $\mathrm{e}$ are the electron mass and charge]

- B-fields $\approx$ several $\times 10^{6}$ Tesla [note: the critical quantum-electrodynamical (vacuum breakdown) field strength is $\mathrm{B}_{\mathrm{c}}=\mathrm{E}_{\mathrm{c}} / \mathrm{c} \approx 10^{10}$ Tesla]

- Ponderomotive Acceleration of Electrons $\approx 10^{17}-10^{30} \mathrm{~g}$ 's $\left(1 \mathrm{~g}=9.81 \mathrm{~m} / \mathrm{sec}^{2}\right)$

- $\quad$ Light Pressure $\approx 10^{9}-10^{15}$ bars

- $\quad$ Plasma Temperatures $>10^{10} \mathrm{~K}$

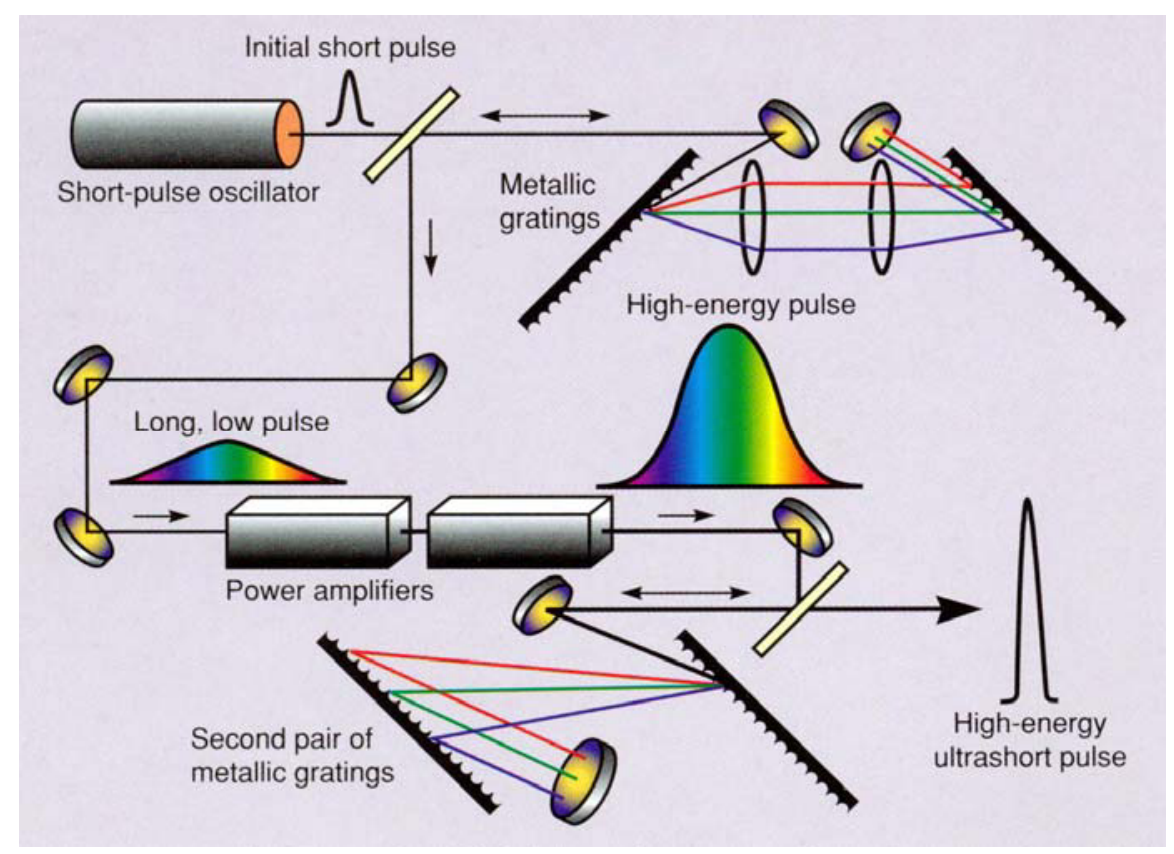

FIGURE 2. Ultrahigh-Intensity Laser (Perry, 1996).

We find from the above data that ultrahigh-intensity lasers can generate an electric field energy density of $\sim 10^{16}-$ $10^{28} \mathrm{~J} / \mathrm{m}^{3}$ and a magnetic field energy density of $\sim 10^{19} \mathrm{~J} / \mathrm{m}^{3}$. These energy densities are about the right order of magnitude to explore generating kilometer to AU sized wormholes. But that would be difficult to engineer on Earth. However, these energy densities are well above what would be required to explore the generation of microwormholes in the lab.

\section{Negative Energy from Squeezed Light}

Negative energy can be generated by an array of ultrahigh intensity (femtosecond) lasers using an ultrafast rotating mirror system. In this scheme (originally due to Davies, 2001) a laser beam is passed through an optical cavity resonator made of lithium niobate $\left(\mathrm{LiNbO}_{3}\right)$ crystal that is shaped like a cylinder with rounded silvered ends to reflect light. The resonator will act to produce a secondary lower frequency light beam in which the pattern of photons is rearranged into pairs. This is the quantum optical squeezing of light effect that we described previously. Therefore, the squeezed light beam emerging from the resonator will contain pulses of negative energy interspersed with pulses of positive energy in accordance with the quantum squeezing model (see Figure 3).

In this example both the negative and positive energy pulses are of $\approx 10^{-15}$ second duration. We could arrange a set of rapidly rotating mirrors to separate the positive and negative energy pulses from each other. The light beam is to strike each mirror surface at a very shallow angle while the rotation ensures that the negative energy pulses are 
reflected at a slightly different angle from the positive energy pulses. A small spatial separation of the two different energy pulses will occur at some distance from the rotating mirror. Another system of mirrors will be needed to redirect the negative energy pulses to an isolated location and concentrate them there.

The rotating mirror system can actually be implemented via non-mechanical means. A chamber of sodium gas is placed within the squeezing cavity, and a laser beam is directed through the gas. The beam is reflected back on itself by a mirror to form a standing wave within the sodium chamber. This wave causes rapid variations in the optical properties of the sodium thus causing rapid variations in the squeezed light so that we can induce rapid reflections of pulses by careful design (Slusher and Yurke, 1986).

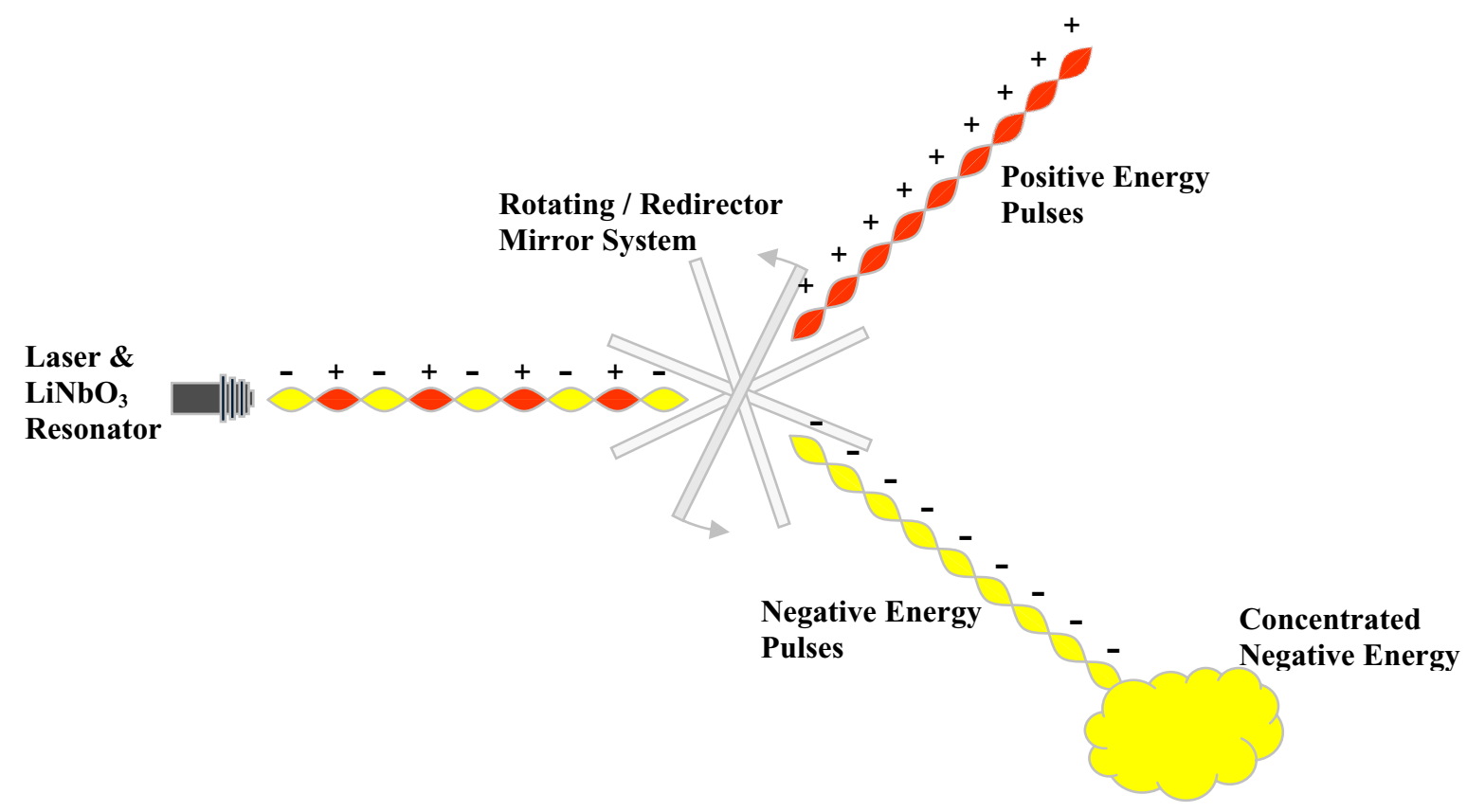

FIGURE 3. Negative Energy Pulses Generated from Quantum Optical Squeezing.

Another way to squeeze light would be to manufacture extremely reliable light pulses containing precisely one, two, three, etc. photons apiece and combine them together to create squeezed states to order. Davies (2001) points out that superimposing many such states could theoretically produce bursts of intense negative energy (see Figure 4). Photonic crystal research has already demonstrated the feasibility of using photonic crystal waveguides (mixing together the classical and quantum properties of optical materials) to engineer light sources that produce beams containing precisely one, two, three, etc., photons. For example, researchers at Melbourne University used a microwave oven to fuse a tiny diamond, just $1 / 1000^{\text {th }}$ of a millimeter, onto an optical fiber, which could be used to create a single photon beam of light (Rabeau et al., 2004a; 2004b). The combining of different beams containing different (finite integer) number of photons is already state-of-the-art practice via numerous optical beam combining methods that can readily be extended to our application.

For the squeezed (electromagnetic) vacuum state, the energy density is given by (Pfenning, 1998):

$$
\begin{aligned}
\rho_{E-s q v a c} & \equiv\left\langle\chi\left|T_{00}\right| \chi\right\rangle \\
& =\left(2 \hbar \omega / L^{3}\right) \sinh r[\sinh r+\cosh r \cos (2 \omega(t-z / c)+\delta)]^{\prime}
\end{aligned}
$$

where $\mathrm{L}^{3}$ is the volume of a large box with sides of length $\mathrm{L}$ (i.e., we put the quantum field in a box with periodic boundary conditions), and $\mathrm{T}_{00}$ is the energy density component of the stress-energy tensor. Equation (2) shows that 
the energy density falls below zero once every cycle when the condition $\cosh r>\sinh r$ is met. It turns out that this is always true for every nonzero value of $r$, so the energy density becomes negative at some point in the cycle for a general squeezed vacuum state. On another note, when a quantum state is close to a squeezed vacuum state, there will almost always be some negative energy densities present, and the fluctuations in $\left\langle\chi\left|\mathrm{T}_{00}\right| \chi\right\rangle$ start to become nearly as large as the expectation value itself.

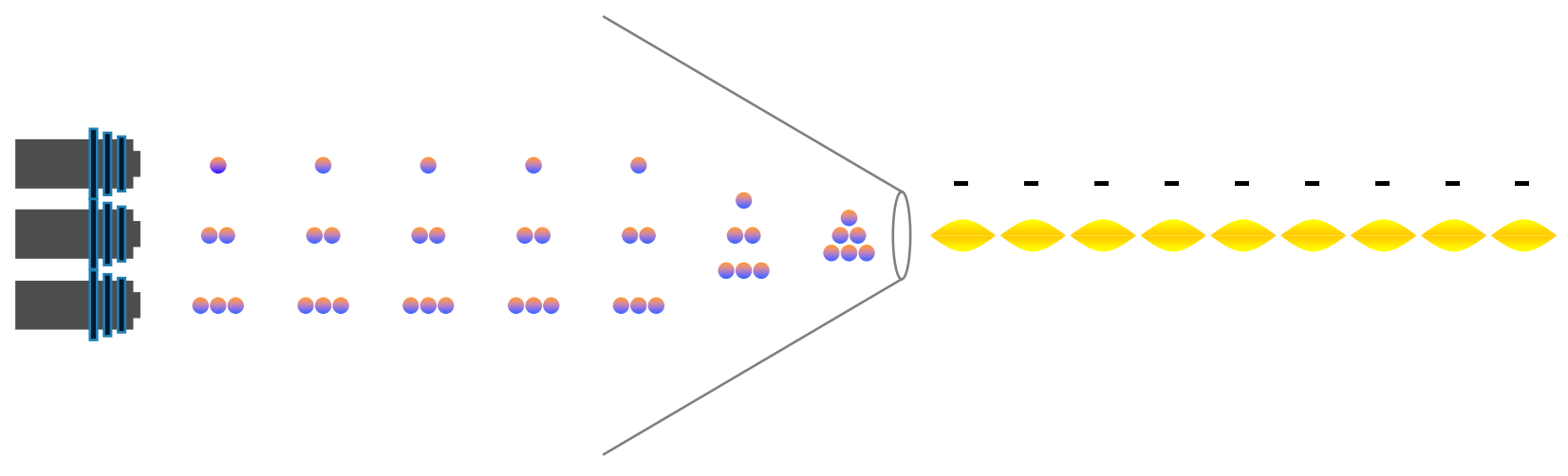

FIGURE 4. Negative Energy Bursts (gold) Generated from Quantum Optical Squeezing.

\section{Observing Negative Energy in the Lab}

Negative energy should be observable in lab experiments. The presence of naturally occurring negative energy regions in space is predicted to produce a unique signature corresponding to lensing, chromaticity and intensity effects in micro- and macro-lensing events on galactic and extragalactic/cosmological scales (Cramer et al., 1995; Torres, Anchordoqui and Romero, 1998; Torres, Romero and Anchordoqui, 1998; Anchordoqui et al., 1999; Safonova, Torres and Romero, 2001; Eiroa, Romero and Torres, 2001). It has been shown that these effects provide a specific signature that allows for discrimination between ordinary (positive mass-energy) and negative massenergy lenses via the spectral analysis of astronomical lensing events. Theoretical modeling of negative energy lensing effects has led to intense astronomical searches for naturally occurring traversable wormholes in the universe (see above references). Computer model simulations and comparison of their results with recent satellite observations of gamma ray bursts (GRBs) has shown that putative negative energy (i.e., traversable wormhole) lensing events very closely resembles the main features of some GRBs. Visser (1997) also points out that current observational data suggests that large amounts of naturally occurring "exotic mass-energy" must have existed sometime between the epoch of galaxy formation and the present in order to (properly) quantitatively account for the "age-of-the-oldest-stars-in-the-galactic halo" problem and the cosmological evolution parameters.

When background light rays strike a negative energy lensing region, they are swept out of the central region thus creating an umbra region of zero intensity. At the edges of the umbra the rays accumulate and create a rainbow-like caustic with enhanced light intensity (see Figure 5). The lensing of a negative mass-energy region is not analogous to a diverging lens because in certain circumstances it can produce more light enhancement than does the lensing of an equivalent positive mass-energy region. Real background sources in lensing events can have non-uniform brightness distributions on their surfaces and a dependency of their emission with the observing frequency. These complications can result in chromaticity effects, i.e. in spectral changes induced by differential lensing during the event. The modeling of such effects is quite lengthy, somewhat model dependent, and with recent application only to astronomical lensing events. Suffice it to say that future work is necessary to scale down the predicted lensing parameters and characterize their effects for lab experiments in which the negative energy will not be of astronomical magnitude. Present ultrahigh-speed optics and optical cavities, lasers, photonic crystal (and switching) technology, sensitive nano-sensor technology, and other techniques are very likely capable of detecting the very small magnitude lensing effects expected in lab experiments. 


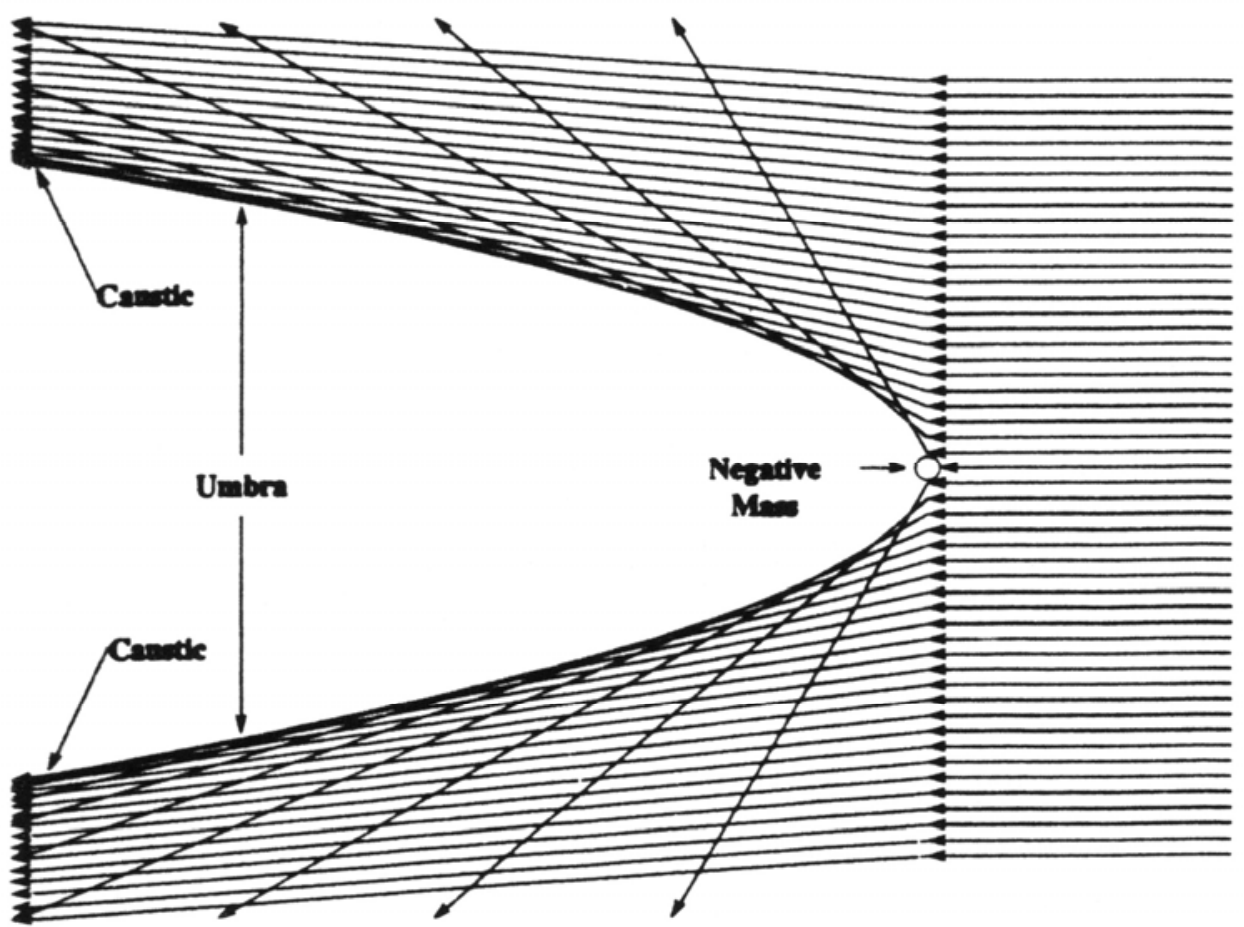

FIGURE 5. Light Deflection by a Negative Mass-Energy Region (Cramer et al., 1995).

\section{The Quantum Inequalities}

The Quantum Inequalities (QI) conjecture is an ad hoc extension of the Heisenberg Uncertainty Principle. They were essentially derived by a small group of curved spacetime quantum field theory specialists for the purpose of making the universe look rational and uninteresting (Pfenning, 1998; see also the excellent review by Ford and Roman, 2003). A small cottage industry of research papers has sprouted up on this one topic alone. The literature is too numerous to cite here. This small group is prejudiced against faster-than-light motion, traversable wormhole and warp drive spacetimes, time machines, negative energy, and other related issues having to do with the violation of the second law of thermodynamics. This group accepts the reality of the theoretical and proven experimental existence of negative energy density and fluxes, but they don't accept the consequences of its various manifestations in spacetime.

The QI supposedly relates the energy density of a free quantum field and the time during which this energy density is observed. When generating negative energy via squeezed quantum states the QI postulates that: a) the longer the pulse of negative energy lasts, the weaker it must be, $b$ ) a pulse of positive energy must follow and the magnitude of the positive pulse must exceed that of the initial negative pulse, c) the longer the time interval between the two pulses, the larger the positive pulse must be. This actually sounds quite reasonable on energy conservation grounds until one finds out that the Casimir Effect (a squeezed vacuum state by virtue of its cavity boundary conditions) violates all three conditions. The Casimir Effect even violates a steady-state QI analysis. Squeezed light was also shown to violate the above postulates. Visser (1997) points out that observational data indicates that large amounts of "exotic mass-energy" is required to exist in the universe in order to account for the observed cosmological evolution parameters. The QI have thus not been verified by laboratory experiments or observational data. The assumptions used to derive the QI and the efficacy of the derivations for various cases has been called into question by numerous investigators. Krasnikov (2004) constructed an explicit counterexample for generalized FTL spacetimes showing that the relevant QI does not hold. Therefore, we will ignore the QI conjecture. 


\section{CONCLUSION}

We reviewed the general relativistic definition of negative energy and identified the forms of negative energy that are known to occur in nature or have been predicted to occur. We examined each of these and characterized their physics and the magnitude of negative energy they are capable of producing. Next we reviewed first-order experimental concepts that we believe will achieve the objective of generating negative energy in the lab. It was demonstrated that radial electric or magnetic fields generated by ultrahigh-intensity lasers are capable of achieving energy densities large enough to observe laboratory-scale spacetime effects. It was also shown that the negative energy densities generated by squeezed quantum state processes are capable of generating negative energy. However, future work is needed to establish the experimental parameters required to specifically separate out the negative energy pulses from the positive energy pulses in optical cavity systems at ultrahigh speed. The astronomical observation of naturally occurring negative energy lensing events has been predicted. The predicted lensing effects have been characterized and quantified but this also needs to be done for laboratory-scale experiments, and this will be the subject of future work. Last, the Quantum Inequalities conjecture is predicted to impose serious constraints on the amount of negative energy that can be generated and utilized. However, this has been disproved to some extent for a variety of spacetimes and quantum field cases. There is also experimental evidence that contradicts the main postulates and assumptions of the QI conjecture.

\section{ACKNOWLEDGMENTS}

We would like to thank the Institute for Advanced Studies at Austin for supporting this work. This work previously originated under Air Force Research Laboratory (AFMC) contract F04611-99-C-0025. We also wish to extend our thanks and appreciation to Frank Mead (AFRL/PRSP, Edwards AFB, CA), members of the Air Staff (Air Force HQ, Pentagon), and Paul Murad (Department of Defense) for encouraging us to explore this topic.

\section{REFERENCES}

Alcubierre, M., “The warp drive: hyper-fast travel within general relativity," Class. Quant. Grav. 11, L73-L77 (1994).

Anchordoqui, L. A., et al., "In Search for Natural Wormholes," Mod. Phys. Lett. A 14, 791-797 (1999).

Barcelo, C., and Visser, M., "Twilight for the energy conditions?," Int. J. Mod. Phys. D 11, 1553 (2002).

Birrell, N. D., and Davies, P. C. W., Quantum fields in curved space, Cambridge University Press, Cambridge, 1984.

Caves, C. M., "Quantum-mechanical noise in an interferometer," Phys. Rev. D 23, 1693-1708 (1981).

Cramer, J. G., et al., "Natural wormholes as gravitational lenses," Phys. Rev. D 51, 3117-3120 (1995).

Davies, P. C. W., "Can moving mirrors violate the second law of thermodynamics?," Phys. Lett. B 11, 215 (1982).

Davies, P. C. W., How to Build a Time Machine, Penguin Books, New York, 2001.

Davis, E. W., "Research Summary Report \#2 to Dr. Hal Puthoff, IASA: Brief Summary of 'Gravitational Vacuum Polarization. Parts I - IV'," NASA Research Center Online Library - Interstellar Studies (1999a).

Davis, E. W., "Research Summary Report \#1 to Dr. Hal Puthoff, IASA: Brief Summary of 'Lorentzian Wormholes from the Gravitationally Squeezed Vacuum,," NASA Research Center Online Library - Interstellar Studies (1999b).

Davis, E. W., Teleportation Physics Study, Final Report AFRL-PR-ED-TR-2003-0034, Air Force Research Laboratory, Air Force Materiel Command, Edwards AFB, CA, 2004, pp. 3-11.

DeWitt, B. S., "Quantum Field Theory in Curved Spacetime,” Phys. Lett. C 19, 295-357 (1975).

Drummond, P. D., and Ficek, Z. (eds.), Quantum Squeezing, Springer-Verlag, Berlin, 2004.

Eiroa, E., Romero, G. E., and Torres, D. F., "Chromaticity Effects in Microlensing by Wormholes," Mod. Phys. Lett. A 16, 973983 (2001).

Epstein, H., Glaser, V., and Jaffe, A., "Nonpositivity of the Energy Density in Quantized Field Theories," Nuovo Cimento 36, 1016-1022 (1965).

Everett, A. E., "Warp drive and causality," Phys. Rev. D 53, 7365-7368 (1996).

Ford, L. H., Proc. Roy. Soc. London A364, 227 (1978).

Ford, L. H., and Roman, T. A., "Negative Energy, Wormholes and Warp Drive," Sci. Am. 13, 84-91 (2003).

Forward, R. L., Alternate Propulsion Energy Sources, Final Report AFRPL TR-83-067, Air Force Rocket Propulsion Laboratory, Air Force Space Tech. Ctr. Space Div., Air Force Systems Command, Edwards AFB, CA, 1983, pp. A1-A14.

Hawking, S. W., and Ellis, G. F. R., The Large-Scale Structure of Space-Time, Cambridge Univ. Press, Cambridge, 1973, pp. 8891, 95-96.

Herrmann, F., “Energy Density and Stress: A New Approach to Teaching Electromagnetism,” Am. J. Phys. 57, 707-714 (1989). 
Hochberg, D., and Kephart, T. W., "Lorentzian wormholes from the gravitationally squeezed vacuum,” Phys. Lett. B 268, 377$383(1991)$.

Krasnikov, S., "Counter example to a quantum inequality," arxiv.org/gr-qc/0409007 (2004).

Milonni, P. W., The Quantum Vacuum: An Introduction to Quantum Electrodynamics, Academic Press, New York, 1994.

Milton, K. A., The Casimir Effect: Physical Manifestations of Zero-Point Energy, World Scientific, New Jersey, 2001.

Morris, M. S., and Thorne, K. S., "Wormholes in spacetime and their use for interstellar travel: A tool for teaching general relativity," Am. J. Phys. 56, 395-412 (1988).

Morris, M. S., Thorne, K. S., and Yurtsever, U., "Wormholes, time machines, and the weak energy conditions," Phys. Rev. Lett. 61, 1446-1449 (1988).

Mourou, G. A., Barty, C. P. J., and Perry, M. D., "Ultrahigh-Intensity Lasers: Physics Of The Extreme On A Tabletop,” Physics Today 51, 22-28 (1998).

Perry, M. D., "Crossing the Petawatt Threshold," Science \& Technology Review (LLNL-DoE publ.), 4-11 (December 1996).

Perry, M. D., "The Amazing Power of the Petawatt," Science \& Technology Review (LLNL-DoE publ.), 4-12 (March 2000).

Pfenning, M. J., Quantum Inequality Restrictions on Negative Energy Densities in Curved Spacetimes, Ph.D. Dissertation, Tufts Univ., Medford, MA, 1998 (arxiv.org/gr-qc/9805037).

Rabeau, J. R., et al., "Fabrication of single nickel-nitrogen defects in diamond by chemical vapor deposition," arxiv.org/condmat/0411245 (2004a).

Rabeau, J. R., et al., "Diamond chemical vapor deposition on optical fibers for fluorescence waveguiding," arxiv.org/condmat/0411249 (2004b).

Robinson, A. L., "Bell Labs Generates Squeezed Light,” Science 230, 927-929 (1985).

Robinson, A. L., "Now Four Laboratories Have Squeezed Light," Science 233, 280-281 (1986).

Safonova, M., Torres, D. F., and Romero, G. E., "Macrolensing Signatures of Large-Scale Violations of the Weak Energy Condition," Mod. Phys. Lett. A 16, 153-162 (2001).

Saunders, S., and Brown, H. R. (eds.), The Philosophy of Vacuum, Clarendon Press, Oxford, 1991.

Slusher, R. E., et al., "Observation of Squeezed States Generated by Four-Wave Mixing in an Optical Cavity," Phys. Rev. Lett. 55, 2409-2412 (1985).

Slusher, R. E., and Yurke, B., "Squeezed Light,” Sci. Am. 254, 50-56 (1986).

Torres, D. F., Anchordoqui, L. A., and Romero, G. E., "Wormholes, Gamma Ray Bursts and the Amount of Negative Mass in the Universe," Mod. Phys. Lett. A 13, 1575-1581 (1998).

Torres, D. F., Romero, G. E., and Anchordoqui, L. A., "Might some gamma ray bursts be an observable signature of natural wormholes," Phys. Rev. D 58, 123001:1-8 (1998).

Visser, M., "Wormholes, baby universes, and causality," Phys. Rev. D 41, 1116-1124 (1990).

Visser, M., Lorentzian Wormholes: From Einstein to Hawking, AIP Press, New York, 1995.

Visser, M., "Energy Conditions in the Epoch of Galaxy Formation," Science 276, 88-90 (1997).

Vollick, D. N., "Negative energy density states for the Dirac field in flat spacetime," Phys. Rev. D 57, 3484-3488 (1998).

Walker, W. R., "Negative energy fluxes and moving mirrors in curved space," Class. Quant. Grav. 2, L37-L40 (1985).

Yu, H., and Shu, W., "Quantum states with negative energy density in the Dirac field and quantum inequalities," Phys. Lett. B 570, 123-128 (2003). 